\title{
Map Estimation for Impingement Cooling with a Fast Extremum Seeking Algorithm
}

\author{
Benjamin Fietzke, Matthias Kiesner, Arne Berthold, \\ Frank Haucke and Rudibert King
}

\begin{abstract}
In many actively controlled processes, such as active flow or combustion control, a set of actuation parameters has to be specified, ranging from actuation frequency to pulse width to geometrical parameters such as actuator spacing. As a specific example, impingement cooling is considered here. Finding the optimal parameters for impingement cooling with steady-state measurements is a time consuming process because of the necessary time to reach thermal equilibrium. This work presents an algorithm for fast extremum seeking to reduce the amount of time needed. It is inspired by an Extremum Seeking Controller, which is a simple but powerful feedback control technique. The first results using this concept are promising, as the magnitude of the optimal pulse frequency for the cooling efficiency of pulsed impingement jets could be found with sufficient precision in a short period of time. The main advantages of this concept are the simple execution on a test rig, its versatility, and the fact that almost no information about the investigated system is necessary.
\end{abstract}

Keywords Impingement cooling $\cdot$ Extremum seeking $\cdot$ Map estimation

B. Fietzke $(\bowtie) \cdot$ M. Kiesner $\cdot$ R. King

Institute of Process and Plant Technology, Technische Universität Berlin,

Chair of Measurement and Control, Hardenbergstr. 36a, 10623 Berlin, Germany

e-mail: b.fietzke@tu-berlin.de

R. King

e-mail: rudibert.king @ tu-berlin.de

\author{
A. Berthold · F. Haucke \\ Institute of Aeronautics and Astronautics, Technische Universität Berlin, \\ Chair of Aerodynamics Marchstr. 12-14, 10587 Berlin, Germany \\ e-mail: arne.berthold@tu-berlin.de \\ F. Haucke \\ e-mail: frank.haucke.1@ @u-berlin.de




\section{Introduction}

The efficiency of gas turbines is steadily being improved by increasing their pressure ratio and the turbine inlet temperature. An increased inlet temperature is acceptable because cooling mechanisms are improved and more resistent blade materials are used, such as superalloys and ceramic thermal barrier coatings [4]. However, despite significant achievements in the field of materials research, additional blade cooling concepts, such as film cooling, are needed for temperatures higher than $1300{ }^{\circ} \mathrm{C}$. In addition to film cooling, impingement cooling inside the turbine blades leads to higher local heat transfer rates compared to conventional convective cooling.

By dynamically forcing the impingement jets, it is possible to further improve the cooling effect via the generation of strong vortex structures that possess increased convective heat transfer capability, which results in higher local convective heat transfer coefficients [7-11, 14].

In this paper, an impingement cooling test rig $[3,5]$ with a $7 \times 7$ impingement array is considered (see Fig. 1). One challenge for an encompassing study is the large number of possible actuation parameters. This results in a time-consuming optimization procedure to find the best combination of parameters. Traditionally, the influence of individual actuation parameters is revealed by steady-state measurements for which it is mandatory to wait until the test rig is in a state of thermal equillibrium. For the specific task considered here, this leads to a measurement time of up to $30 \mathrm{~min}$ for each possible combination of actuation parameters. Therefore, an alternative method
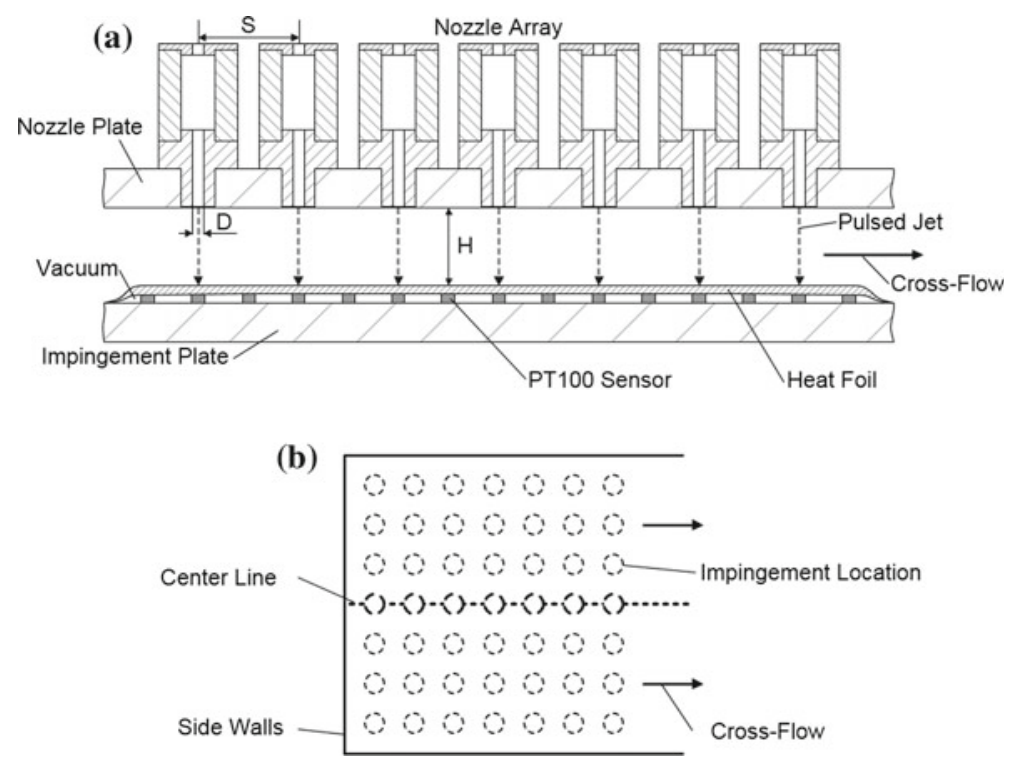

Fig. 1 a Schematic of the experimental setup. b Impingement locations with side walls and induced cross-flow from the top. Reproduced from [3] 
is proposed that makes it possible to locate extrema in a shorter period of time. The presented concept is inspired by a well-known closed-loop controller, namely the Extremum Seeking Controller (ESC). Generally, an ESC drives a system to an optimal operating point without much knowledge about the system. More specifically, no mathematical model of the process is required but just the knowledge that an extremum exists. However, some estimate of its response time must be known [2]. A closed-loop ESC will find the next local optimum in a steady-state map of a system and will remain in its vicinity. While this suffices for many applications, the goal of the present contribution is to produce an estimate of the complete map, possibly comprising several local extrema. Fewer precise steady-state measurements near the located optima can then be performed to obtain detailed information about the best heat transfer conditions.

In this work, this idea will be exploited to find a relationship between the actuation parameters of a pulsed jet and the realized heat transfer coefficients. More specifically, the optimal actuation frequency $f$ of the pulsed jets and its impact on the cooling effect is studied.

The paper is organized as follows. Section 2 introduces the experimental setup. The concept of using an ESC-like algorithm in fast map estimation is detailed in Sect. 3. Results are presented in Sect. 4, and conclusions are drawn in Sect. 5.

\section{Experimental Setup}

The experimental setup for this work is based on previous investigations by Haucke and Berthold [3, 5]. A schematic of the setup is given in Fig. 1a. As shown, the nozzles are arranged in a $7 \times 7$ inline array on a nozzle plate. The nozzles have an exit diameter of $D=12 \mathrm{~mm}$ and a distance of $S=60 \mathrm{~mm}$ to the next nozzles. The impingement distance between the nozzles and the target plate is $H=36 \mathrm{~mm}$. The target plate consists of a thin steel foil $(600 \times 600 \times 0.05 \mathrm{~mm})$ and a wooden impingement plate $(1 \mathrm{~m} \times 1 \mathrm{~m} \times 12 \mathrm{~mm})$. Three sides of the foil are enclosed by side walls that induce a cross-flow, which superimposes the pulsed jets and therefore reduces the efficiency of the impingement cooling.

The edges of the foil are sealed, and a vacuum is applied to press the heat foil to the impingement plate. The steel foil itself is connected to a constant current power source. Because the foil being a resistor, it serves as a heat source for the setup.

Under the center line of the impingement jets (Fig. 1b) and between the heat foil and the impingement plate, 15 Pt100 temperature sensors are integrated to measure the temperature distribution in a straight line. For each jet, a Pt100 sensor is placed under the center of the impingement location. Additional sensors are mounted in the middle between two adjacent jets. ${ }^{1}$

The precision of the class A Pt100 sensors is based on DIN EN 60751. Therefore, the measurement error of the sensors is lower than $0.25^{\circ} \mathrm{C}$ in the temperature range $T_{i}=\left\{20^{\circ} \mathrm{C} \ldots 40^{\circ} \mathrm{C}\right\}$ considered here. Additionally, a Pt100 sensor is placed in

\footnotetext{
${ }^{1}$ Thermocouples instead of Pt100 sensors would allow for an even higher bandwidth if necessary.
} 
Fig. 2 Setup of a valve-nozzle combination in the $7 \times 7$ nozzle array [3]

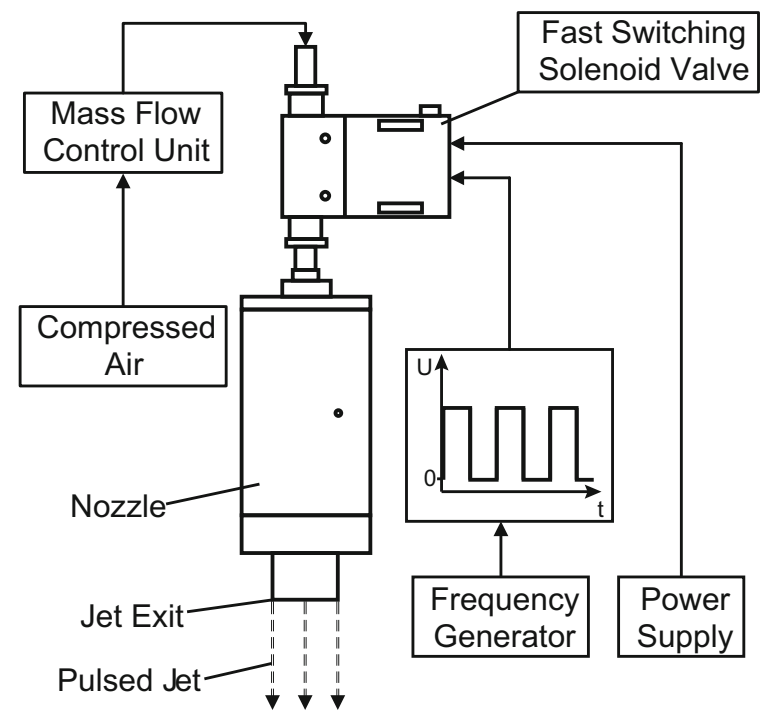

the center nozzle to measure the temperature of the cooling air, $T_{\text {jet }}(t)$. In $[3,5]$, a thermography liquid crystal (TLC) foil is used for temperature measurements on the plate. Because measuring with a TLC foil is based on the evaluation of image analytical data, it is more difficult to obtain information about transient behavior.

To generate pulsed jets, every nozzle is equipped with a fast switching solenoid valve (FESTO MHJ9-QS-4-MF), as shown in Fig. 2. The permissible maximum normalized volume flow of the valves is $V_{\mathrm{N}}=1601_{\mathrm{N}} / \mathrm{min}$, and the maximum state switching frequency is $f_{\text {st, } \max }=1000 \mathrm{~Hz}$. The air mass flow is supplied by an inhouse compressor. A mass flow control unit is plugged in between each of the seven lines of valves and the compressor. The control unit adjusts the desired amount of cooling air and directs it to an air divider. The latter splits the flow equally into seven smaller flows for the corresponding valve line. In this setup, the operating point for the mass flow control unit is set so that the resulting averaged Reynolds number for each nozzle is $\bar{R} e \approx 7200$, calculated with respect to the mean jet velocity. The frequency of the pulsed jets is determined by a square wave signal of an Field Programmable Gate Array (FPGA) frequency generator, making it possible to precisely change the frequency with a high resolution whereby the valves open and close dynamically with a duty cycle of $50 \%$.

\section{Concept}

As mentioned, the applied concept for the fast map estimation is inspired by the basic ESC algorithm, which has been successfully implemented in various systems $[6,13]$ and which will be described in some detail below. The main advantage of 
the ESC is the possibility of driving a dynamic system to an optimal operating point despite the fact that no further information is needed besides a rough estimate of the dominant time constant of the system. This will now be exploited in an openloop manner to estimate the gradient of an unknown map. Thus, local extrema can be found. To keep the contribution compact, the explanation will be built on the task of increasing cooling efficiency, which is investigated in this paper, instead of separate treatments of the general and specific cases. Cooling efficiency depends on the actuation frequency $f$ of the pulsed jets, that is, an optimal value for this actuation frequency is sought.

As an output of the considered system, an average temperature difference $\Delta T(t)=$ $\bar{T}(t)-T_{\text {jet }}(t)$ is introduced. Here, $\bar{T}(t)$ is the arithmetic mean of the Pt100 measurements (see Sect.2), while $T_{\text {jet }}(t)$ is the temperature of the cooling jet. For a steady state, this difference is denoted by $\Delta T_{\mathrm{s}}$.

The central idea of ESC is explained in Fig. 3. Assume that the system input $f(t)$ is on the left of the map's minimum and $f(t)$ is perturbed by $a_{\mathrm{f}} \sin (\omega t)$ with the perturbation amplitude $a_{\mathrm{f}}$ and the perturbation frequency $\omega$. The output $\Delta T_{\mathrm{s}}$ then has a shape similar to a negative sine wave, which means a phase shift of $180^{\circ}$ in comparison to the perturbation $a_{\mathrm{f}} \sin (\omega t)$. The amplitude of the output is roughly the input amplitude $a_{\mathrm{f}}$ multiplied by the unknown local gradient of the map, $\mathrm{d}\left(\Delta T_{\mathrm{s}}\right) / \mathrm{d} f$. However, if an input is applied to the right of the minimum, the same input signal would cause an output signal similar to a positive sine wave, that is, with a zero phase shift. Therefore, due to a harmonic input perturbation, an output phase shift yields information whether a minimum exists towards lower or larger input values.

In a classical (closed-loop) ESC, the output signal would be further processed to obtain an estimate of the size of the slope of the map to then apply a gradientbased optimization that drives the input to the optimal value. For this, the excitation frequency has to be small enough to obtain a steady-state input-output relationship

Fig. 3 Phase switch of the output $\Delta T_{\mathrm{s}}$ in a steady-state map when moving across an extremum

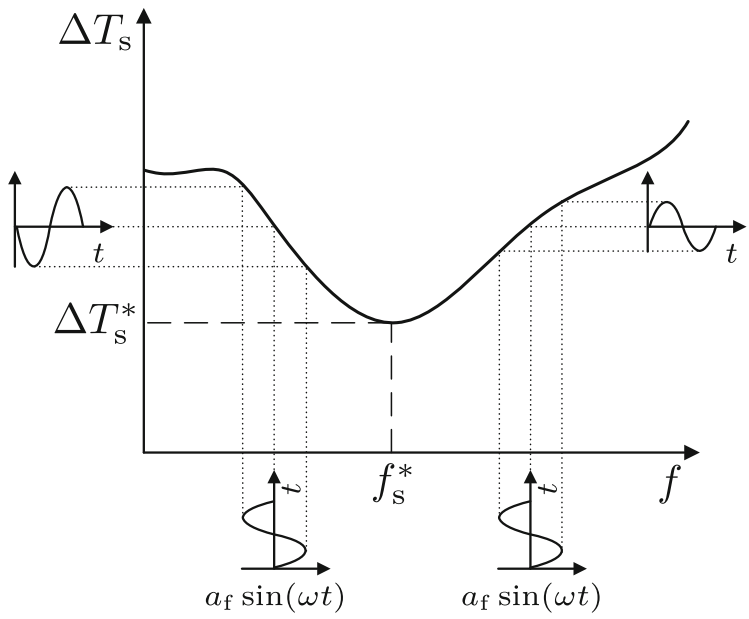


[2]. In the case considered here, the loop will not be closed, but the input $f$ will be continiously increased in addition to the harmonic perturbation to scan the complete input range. Moreover, to speed up evaluation, this scan will not be performed in a quasi-steady manner so that dynamic effects will appear. That is, additional, unwanted phase shifts between the input and output will be observed.

The concept for fast map estimation is sketched in Fig. 4. The process itself can either be described by its steady-state map or by a block in the corresponding block diagram representing the dynamic behavior of the system (see Fig.4). Note that both blocks are unknown. In this paper, the steady-state map characterizes the gain from the input signal, which is the pulse frequency $f(t)$ of the jets, to the average temperature difference $\Delta T(t)$. When the corresponding thermal equillibrium $\bar{T}_{\mathrm{S}}$ is reached, it results in a steady temperature difference $\Delta T_{\mathrm{s}}$.

To generate the input, the on-off solenoid valves are operated periodically with a frequency $f(t)$ that is ramped up $\left(m_{\mathrm{f}} t\right)$ and perturbed harmonically, that is, the excitation frequency reads as follows:

$$
f(t)=a_{\mathrm{f}} \sin (\omega t)+m_{\mathrm{f}} t .
$$

Now, as a first-order approximation, the output of the process $\Delta T(t)$ can be described as:

$$
\Delta T(t) \approx \Delta T_{\mathrm{s}}+a_{\mathrm{f}} \frac{\mathrm{d}\left(\Delta T_{\mathrm{s}}\right)}{\mathrm{d} f} \sin (\omega t) .
$$

If the map was linear and the excitation of the system quasi-steady, equality would result in Eq. (2). The measured temperature difference $\Delta T(t)$ is then bandpass (BP)filtered with zero phase shift to cut off the steady part $\Delta T_{\mathrm{s}}$. For that, a fourth-order

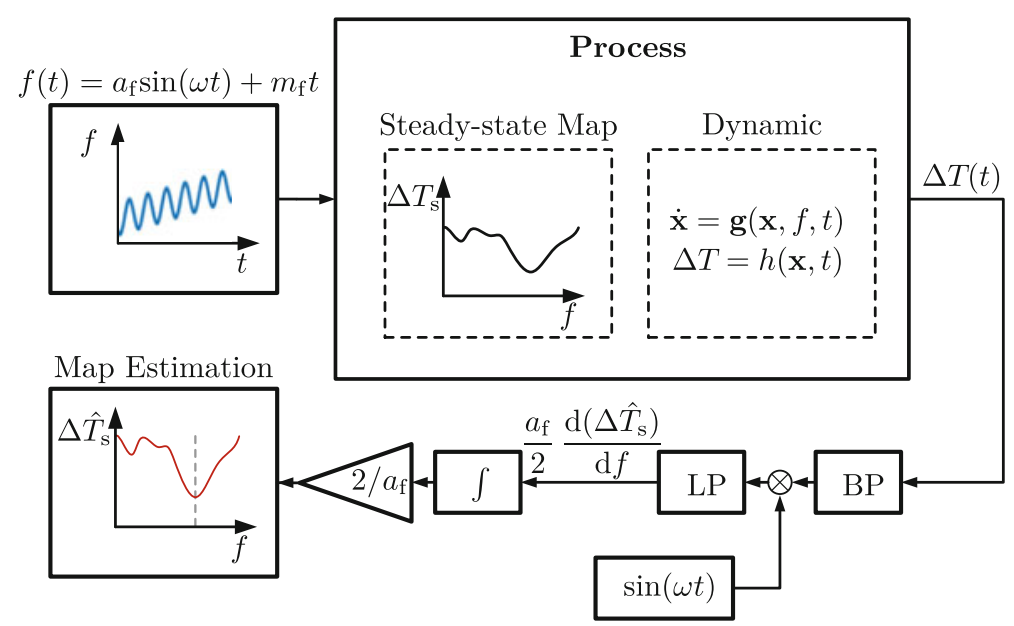

Fig. 4 Concept of fast open-loop extremum seeking 
BP filter with identical upper and lower corner frequencies equal to the perturbation frequency $\omega$ is applied, as in:

$$
G_{\mathrm{BP}}(s)=\left(\frac{2 \omega s}{(s+\omega)^{2}}\right)^{2},
$$

where $s$ is the Laplace variable. A fourth-order BP is chosen to obtain a larger roll-off of its gain for other frequencies. For zero initial conditions, the BP-filtered data can be described as follows:

$$
\Delta T_{\mathrm{BP}}(t) \approx a_{\mathrm{f}} \frac{\mathrm{d}\left(\Delta T_{\mathrm{s}}\right)}{\mathrm{d} f} \sin (\omega t) .
$$

After this step, $\Delta T_{\mathrm{BP}}(t)$ is demodulated with the perturbation signal. The demodulated signal $\Delta T_{\text {Dem }}(t)$ can be described with the following expression, where the first term on the right-hand side will vary with time when the actuation frequency is ramped up:

$$
\Delta T_{\mathrm{Dem}}(t)=\sin (\omega t) \Delta T_{\mathrm{BP}}(t) \approx \underbrace{\frac{a_{\mathrm{f}}}{2} \frac{\mathrm{d}\left(\Delta T_{\mathrm{s}}\right)}{\mathrm{d} f}}_{\text {non-periodic }}-\underbrace{\frac{a_{\mathrm{f}}}{2} \frac{\mathrm{d}\left(\Delta T_{\mathrm{s}}\right)}{\mathrm{d} f} \cos (2 \omega t)}_{\text {periodic }} .
$$

After the demodulation, the signal has a non-periodic and a periodic part with doubled frequency. The latter can be filtered out with a lowpass (LP) filter, such as a Butterworth filter. The outcome of filtering can be improved by using an acausal filter, as all calculations are performed after the experiment is finished. As a result, the LP output $\Delta T_{\mathrm{LP}}(t)$ mainly consists of the non-periodic part

$$
\Delta T_{\mathrm{LP}}(t) \approx \frac{a_{\mathrm{f}}}{2} \frac{\mathrm{d}\left(\Delta T_{\mathrm{s}}\right)}{\mathrm{d} f} .
$$

The factor $a_{\mathrm{f}} / 2$ can be removed easily by proper scaling. To finally obtain an estimate $\Delta \hat{T}_{\mathrm{s}}=\Delta \hat{T}_{\mathrm{s}}(f)$, the gradient $\mathrm{d}\left(\Delta T_{\mathrm{s}}\right) / \mathrm{d} f$ must be integrated with respect to the frequency $f$. In the experiment, however, only an integration over time is possible. To that end,

$$
\Delta \hat{T}_{\mathrm{s}}(f)=\frac{2}{a_{\mathrm{f}}} \int \Delta T_{\mathrm{LP}} \mathrm{d} f \approx \int \frac{\mathrm{d}\left(\Delta T_{\mathrm{s}}\right)}{\mathrm{d} f} \mathrm{~d} f
$$

is reformulated as

$$
\Delta \hat{T}_{\mathrm{s}}(f) \approx \int \frac{\mathrm{d}\left(\Delta T_{\mathrm{s}}\right)}{\mathrm{d} f} \frac{\mathrm{d} f}{\mathrm{~d} t} \mathrm{~d} t
$$


The time derivative of $f$ contains a constant and a fast harmonic part (see Eq. (1)). The latter was only introduced to obtain fast local gradient information and leads to high-frequency oscillation of the integrated result. Therefore, only the slope of the $\operatorname{ramp} m_{\mathrm{f}}$ is used for the time derivative. Hence,

$$
\Delta \hat{T}_{\mathrm{s}}(f) \approx m_{\mathrm{f}} \int \frac{\mathrm{d}\left(\Delta T_{\mathrm{s}}\right)}{\mathrm{d} f} \mathrm{~d} t .
$$

is obtained. Low values of the estimated temperature difference $\Delta \hat{T}_{\mathrm{s}}(f)$ between the cooling jets and the heated foil indicate actuation frequencies resulting in a large heat transfer.

\section{Results}

At the start of every measurement series, the frequency of the pulsed jets is set to a constant value of $200 \mathrm{~Hz}$ to reach steady-state. The ramp then starts and continues until $f=1000 \mathrm{~Hz}$ is reached. In Fig. 5, an example with $a_{\mathrm{f}}=20 \mathrm{~Hz}, \omega=0.2 \mathrm{rad} / \mathrm{s}$, and a ramp duration of $30 \mathrm{~min}$, that is, $m_{\mathrm{f}}=0 . \overline{4} \mathrm{~Hz} / \mathrm{s}$, is displayed. The measured unsteady temperature of the cooling air $T_{\text {jet }}(t)$ is shown in panel $\mathrm{b}$ ). To decrease the influence of this unwanted dynamic, $T_{\text {jet }}(t)$ is LP-filtered, resulting in smoother progress. In addition, the plate's mean temperature $\bar{T}(t)$ is displayed. To evaluate the cooling efficiency, the temperature difference between $\bar{T}(t)$ and the filtered data $T_{\text {jet,LP }}(t)$ is used:

$$
\Delta T(t)=\bar{T}(t)-T_{\mathrm{jet}, \mathrm{LP}}(t)
$$

This is shown at the bottom of Fig. 5. The lowest difference is seen for $t \approx 1550 \mathrm{~s}$ when $f \approx 770 \mathrm{~Hz}$. However, this is not the optimal frequency, as will be shown below.

As mentioned in the previous section and as a result of the non-quasi-steady excitation, the output signal $\Delta T(t)$ lags with respect to the input signal $f(t)$ due to the dynamic behavior of the process. Additionally, this lag depends on whether the process is left or right of an extremum (see discussion of Fig. 3). This can be illustrated by comparing the perturbation $\sin (\omega t)$ with $\Delta T(t)$ or, as the BP features a zero phase shift, with the normalized output signal of the BP $\Delta \tilde{T}_{\mathrm{BP}}(t)=\Delta T_{\mathrm{BP}}(t) / \max \left(\Delta T_{\mathrm{BP}}(t)\right)$, as done at the top of Fig. 6 . Note that in this Figure, $\mathrm{t}=0$ marks the start of the sine ramp, so the first $\sim 200 \mathrm{~s}$ to reach steady-state are cut off.

Assuming the process is left of a minimum of the unknown map, then, according to Fig. 3, the temporal minimum of the output signal should coincide with a temporal maximum of the input. As this is not exactly the case in the first part of Fig. 6 (up to 
Fig. 5 a Ramp of sinusoidal frequency with $a_{\mathrm{f}}=20 \mathrm{~Hz}$ and $\omega=0.2 \mathrm{rad} / \mathrm{s}$ starting at $200 \mathrm{~Hz}$ and reaching $1000 \mathrm{~Hz}$ in $30 \mathrm{~min}$. Waiting times at both the beginning and the end are introduced as well. b Temperature of the cooling air, original and LP-filtered. c Averaged temperature of the heat foil's center line. d Difference between averaged temperature $\bar{T}(t)$ and LP-filtered jet temperature $T_{\text {jet,LP }}(t)$

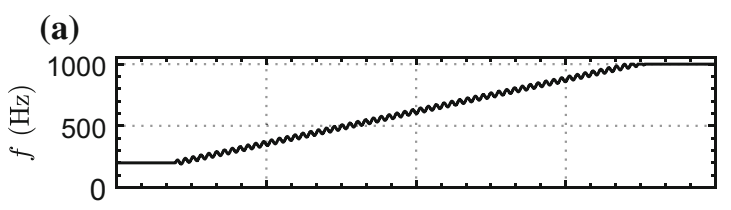

(b)

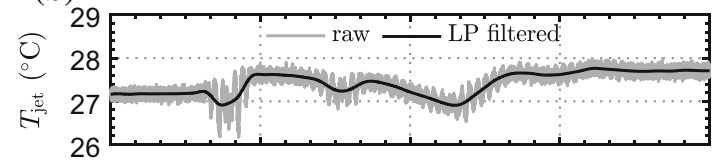

(c)

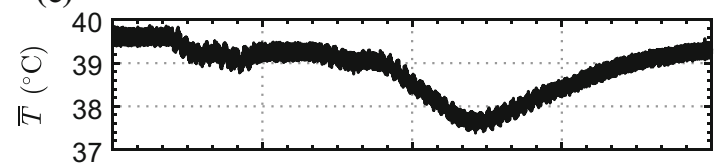

(d)

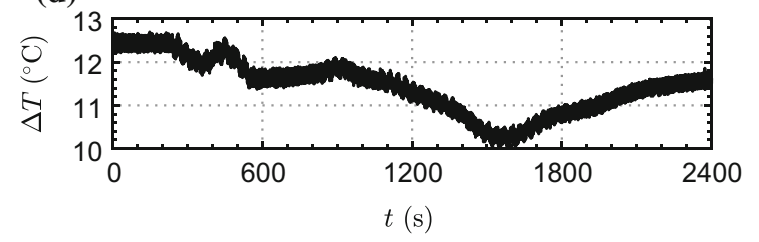

$90 \mathrm{~s})$, the difference can be attributed to the unknown phase shift caused by the slow response of $\Delta T(t)$.

However, for the experiment shown in Fig. 6, it is obvious that the process moves to the right of the minimum somewhere in the time span from 90 to $100 \mathrm{~s}$, as, following the arguments for Fig.3, a maximal output value is now near a maximal input value. As a result, estimating the phase shift would require the information from the map, which is not available at this point. To resolve the problem, the phase shift is determined from squared signals:

$$
\begin{aligned}
& \alpha=\sin (\omega t)^{2} \\
& \beta=\Delta T_{\mathrm{BP}}^{2}(t),
\end{aligned}
$$

as now both the maxima and minima of the signal will result in a maximum. The phase shift can now easily be estimated for those time frames where the process crosses an extremum on the map. To that end, the cross-correlation $R_{\alpha, \beta}(\tau)$ between $\alpha$ and $\beta$ is calculated, where $R_{\alpha, \beta}(\tau)$ is a function of the time shift variable $\tau$. The maximum value of $R_{\alpha, \beta}(\tau)$ determines the average time shift $\tau_{\text {sh }}$ of the process. Since $\alpha$ is periodic, only a frame of the period length of $\alpha$ is considered for this determination:

$$
\tilde{R}_{\alpha, \beta}(\tau)=\frac{R_{\alpha, \beta}(\tau)}{R_{\alpha, \beta}(0)} .
$$


Fig. 6 Top: Phase comparison between the perturbation and the normalized BP output. Bottom: Phase comparison after phase correction. $t=0$ describes the start of the sine ramp
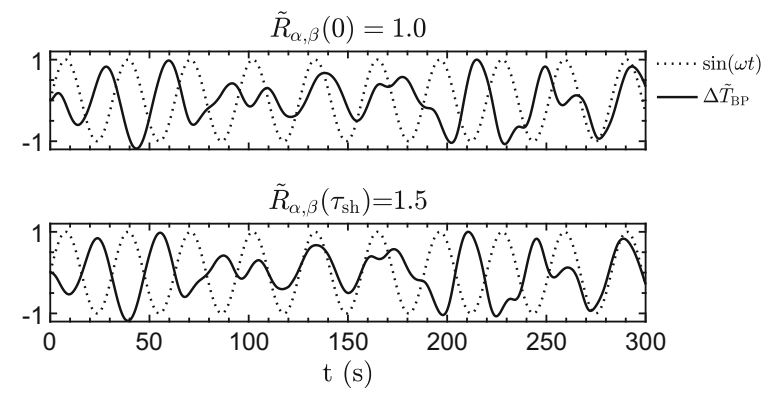

At the bottom of Fig. 6, the phase-corrected signal, obtained with this procedure, is displayed. The extrema of the phase-corrected signal are better aligned with the sine extrema. $\tilde{R}_{\alpha, \beta}\left(\tau_{\mathrm{sh}}\right)$ is around $50 \%$ higher than before phase correction. Comparing minimum-minimum and minimum-maximum pairs in the time traces, the process seems to be left of a map's local minimum for $0<t<90 \mathrm{~s}$ and $190<t<250 \mathrm{~s}$ but right of a map's local minimum for $100<t<160 \mathrm{~s}$ and $260<t<300 \mathrm{~s}$.

The results of the map estimation exploring the ESC algorithm are displayed in the upper panel of Fig. 7. Estimated maps are shown with three different perturbation frequencies $\omega$, a perturbation amplitude $a_{\mathrm{f}}=20 \mathrm{~Hz}$, and a sine ramp duration of $30 \mathrm{~min}$. Note that the map is normalized:

$$
\Delta \hat{\tilde{T}}_{\mathrm{s}}=\frac{\Delta \hat{T}_{\mathrm{s}}}{\Delta \hat{T}_{\mathrm{s}}(f=200 \mathrm{~Hz})} .
$$

For all three settings, the location of the estimated optimum is near $700 \mathrm{~Hz}$, which is significantly different from the apparent optimum in Fig. 5; this is likely due to dynamic effects. It must be pointed out that, taking the waiting time before and after the ramp into account, each of these experiments lasted around $40 \mathrm{~min}$. For comparison, at the bottom of Fig. 7, the averaged and normalized Nusselt number $\tilde{N} u_{\text {av }}{ }^{2}$ of the impingement test rig is given. In general, the Nusselt number characterizes the relationship between convective and conductive heat transfer. The data for the Nusselt number determination is obtained with the same impingement parameters but using a TLC foil and a camera system for temperature measurement ([3] for more details). The determination of each individual data point lasted $30 \mathrm{~min}$ in the steady-state approach, that is, on the coarse measurement grid, the expenditure of time increased by a factor of almost 7 .

Compared to the estimated optimal frequency obtained through ESC map estimation, the optimal frequency in the Nusselt number map is nearly the same. The minimum of the temperature difference $\Delta T_{\mathrm{S}}(t)$ between the steel foil and the cooling air should always go hand in hand with the maximum of the Nusselt number.

\footnotetext{
${ }^{2}$ To obtain a normalization, the calculated Nusselt number is divided by the Nusselt number determined from an experiment with steady, non-pulsed impingement jets. In both the steady and the non-steady cases, identical average massflow rates are used.
} 
Fig. 7 Top: Estimated steady-state map for different perturbation frequencies. Bottom: Nusselt number of the heat transfer for impingement cooling calculated based on steady-state measurements
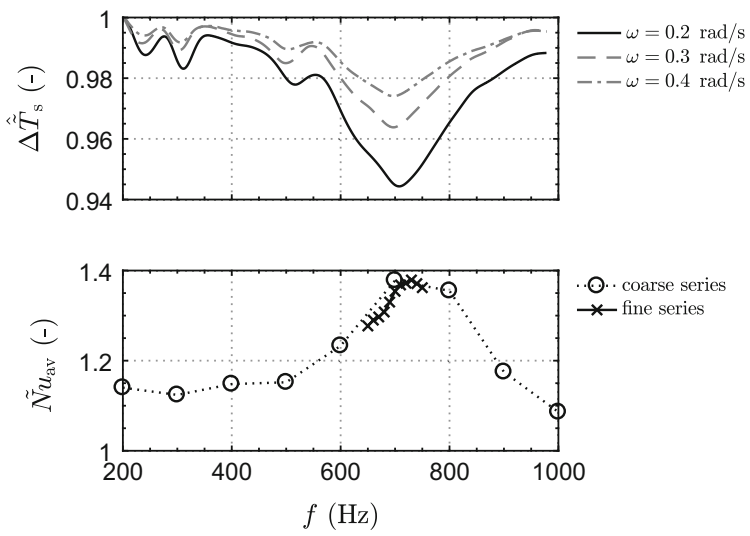

As crossing any extremum in the map results in a phase shift of $180^{\circ}$, such extrema with respect to the actuation frequency are likely to have rather high precision. However, $\Delta \hat{T}_{\mathrm{s}}$ has to be obtained from the integration of a noisy gradient. Therefore, this estimate is deemed rather uncertain.

\section{Conclusion}

Adapting the ESC algorithm for open-loop fast map estimation results in a concept that delivers promising estimates of the locations of extrema on a steady-state map. The big advantage of this concept is the simple execution on the test rig and a robustness to a variation of the algorithm's parameters. Moreover, there are few requirements with respect to the knowledge about the system under study.

Three different perturbation frequencies were tested. In all cases, optima were found near an optimum that was confirmed by time-consuming steady-state measurements. An increase in the sine ramp duration from 30 to $45 \mathrm{~min}$ resulted in no significant differences in the found locations. In contrast, there is no indication that a sine ramp with a shorter duration than $30 \mathrm{~min}$ would result in significantly different locations. Hence, a ramp with shorter duration could further increase the time-saving potential. This will be part of future studies.

Even with a 30-min ramp, the saving of time is crucial compared to steady-state measurements using a TLC foil. While in steady-state measurements a measurement point needs around $30 \mathrm{~min}$, it is possible to scan the whole frequency range with one sine ramp at the same time. As the ESC approach only yields an estimate, its results can be used in a second step to precisely characterize an optimum with the classical approach, as was done here with a fine steady-state measurement grid (see Fig. 7). Another promising idea is to extend the concept for a multidimensional parameter variation, as has already been done in terms of closed-loop ESC [1, 2, 12]. 
Acknowledgements The authors gratefully acknowledge the support of the Deutsche Forschungsgemeinschaft (DFG) as part of the Collaborative Research Center CRC 1029 "Substantial efficiency increase in gas turbines through direct use of coupled unsteady combustion and flow dynamics" in projects B03 and B06. Special thanks go to Joachim Kraatz and Klaus Noack who helped with the electrical setup for the measurements.

\section{References}

1. Ariyur, K.B., Krstic, M.: Analysis and design of multivariable extremum seeking. Am. Control Conf. (2002). https://doi.org/10.1109/ACC.2002.1025231

2. Ariyur, K.B., Krstic, M.: Real-Time Optimization by Extremum-Seeking Control. Wiley (2003)

3. Berthold, A., Haucke, F.: Experimental investigation of dynamically forced impingement cooling. ASME Turbo Expo (2017). https://doi.org/10.1115/GT2017-63140

4. Clarke, D.R., Oechsner, M., Padture, N.P.: Thermal-barrier coatings fore more efficient gasturbine engines. MRS Bull. 37(10), 891-898 (2012)

5. Haucke, F., Nitsche, W., Peitsch, D.: Enhanced convective heat transfer due to dynamically forced impingement jet array. ASME Turbo Expo (2016). https://doi.org/10.1115/GT201657360

6. Henning, L., Becker, R., Feuerbach, G., Muminovic, R., King, R., Brunn, A., Nitsche, W.: Extensions of adaptive slope-seeking for active flow control. Proc. Inst. Mech. Eng. Part I: J. Syst. Control Eng. 222(5), 309-322 (2008)

7. Herwig, H., Middelberg, G.: The physics of unsteady jet impingement and its heat transfer performance. Acta Mech. 201, 171-184 (2008)

8. Hofmann, H.M., Movileanu, D.L., Kind, M., Martin, H.: Influence of a pulsation on heat transfer and flow structure in submerged impinging jets. Int. J. Heat Mass Transf. 50, 3638-3648 (2007)

9. Janetzke, T.: Experimental investigations of flow field and heat transfer characteristics due to periodically pulsating impinging air jets. J. Heat Mass Transf. 45, 193-206 (1998)

10. Liu, T., Sullivan, J.P.: Heat transfer and flow structures in an excited circular impingement jet. J. Heat Mass Transf. 39, 3695-3706 (1996)

11. Middelberg, G., Herwig, H.: Convective heat transfer under unsteady impinging jets: The effect of the shape of the unsteadiness. J. Heat Mass Transf. 45, 1519-1532 (2009)

12. Rotea, M.A.: Analysis of multivariable extremum seeking algorithms. Am. Control Conf. (2000). https://doi.org/10.1109/ACC.2000.878937

13. Tan, Y., Moase, W.H., Manzie, C., Nešić, D., Mareels, I.M.Y.: Extremum seeking from 1922 to 2010. In: Chinese Control Conference (CCC), pp. 14-26 (2010)

14. Vejrazka, J., Tihon, J., Marty, Ph., Sobolik, V.: Effect of an external excitation on the flow structure in a circular impinging jet. Phys. Fluids 17, 105102-01-14 (2005) 